\title{
Energy based Methods for Medical Image Segmentation
}

\author{
Pamela Juneja \\ SISTec \\ Bhopal,India
}

\author{
Ramgopal Kashyap \\ SISTec \\ Bhopal,India
}

\begin{abstract}
Health care applications have become boon for the healthcare industry. It needs correct segmentation connected with medical images regarding correct diagnosis. An efficient method assures good quality segmentation of medical images. Segmentation methods are classified as edge based, region based, clustering based, Level set methods (LSM) and Energy based methods. In this paper, a survey on all the effective methods those are capable for accurate segmentation is given, however quick process employing correct segments is still difficult. Some existing methods do correction and some badly pertain to deep irregularity in images. The wide range of the problems of computer vision may make good use of image segmentation. This paper studies and evaluate the different methods for segmentation techniques. This study is useful for determining the appropriate use of the image segmentation methods and for improving their accuracy and performance and also works on the main objective, which is designing new algorithms. The main goal is to make the image more simple and meaningful. After a brief description of each method an experimental comparison of some empirical (goodness and discrepancy) methods commonly used is then given to provide a rank of their evaluation abilities. This study is helpful for an appropriate use of existing segmentation methods and for improving their performance as well as for systematically designing new segmentation methods.
\end{abstract}

\section{Keywords}

Active contour models,Clustering based segmentation,Energy based method,Hybrid method, Intensity irregularity, Level set method,Local image fitting, Local binary fitting, Medical image segmentation, Region Based Segmentation.

\section{INTRODUCTION}

The purpose of image segmentation is to partition an image into meaningful regions with respect to a particular application. Image segmentation, is often an essential step in image analysis, object representation, visualization, and many other image processing tasks. The segmentation is based on measurements taken from the image and might be gray level, color, texture, depth or motion. Segmentation divides an image into its constituent regions or objects. Segmentation of images is a difficult task in image processing. Segmentation allows in extracting the objects in images. Image segmentation is an important image processing technique that splits the image into sub parts and extracts the object of interest by dividing the pixels based on characteristics like gray level, intensity, texture, etc. Its main aim is to differentiate object and background in an image[1]. Digital image processing consists of a number of steps like image capturing, preprocessing, segmentation, representation, description, recognition and interpretation.Segmentation is one of the difficult steps as the success of later steps relies heavily on the result of this step. Segmentation assigns a label to every pixel in the image. The output of segmentation process can be a contour extracted from an image or set of various segments that covers the whole of the image.
Segmentation has wide application areas like Machine Vision[2].This technology provides image based automatic processing for applications like robot guidance, packaging inspection, wood quality inspection, etc., Content based image retrieval[3] used to search images from large databases based on content analysis rather than metadata. It matches contents based data like color, shapes, texture, etc. while searching for images, medical images[4] Locating Tumors, for treatment planning, Surgery planning, etc., face detection, pedestrian detection systems used in intelligent video surveillance systems etc., Fingerprint and Iris recognition, traffic control systems etc. Image segmentation is nowadays widely used in medical field. Segmentation helps to analyze the medical images to detect various diseases or affected areas in a human body like detecting a brain tumor or any cancerous growth in internal organs of the human body. It also helps to study the anatomy of the human bodythat helps in treatment planning and also used for virtual surgery simulation.Segmentation algorithms are based on one of two basic properties of color, gray values, or texture: discontinuity and similarity. The first category is to partition an image based on abrupt changes in intensity, such as edges in an image. Second category is based on partitioning an image into regions that are similar, according to predefined criteria. Histogram thresholding approach falls under this category. Usually image segmentation is an initial and vital step in a series of processes aimed at overall image understanding [5].

\section{IMAGE SEGMENTATION METHODS CLASSIFICATION}

It is a middle level processing technique that is used to divide an image into several parts by grouping the pixels to form a homogeneous region on the basis of gray level, color, texture and intensity. The main aim of segmentation is to get more information about the image to make image annotation easier. It also clearly differentiates an image foreground from background [6]. Many image segmentation techniques have been developed by researchers and scientists, some of the most important and widely used image segmentation techniques are shown in Fig.1. Latest research work on image segmentation techniques highlighted in Fig.1 is discussed and evaluated below.

\subsection{Discontinuity based methods:}

The discontinuity based methods are based on the principle of variation of intensity of pixels in an image. There will be significant changes in intensity levels among neighboring pixels and therefore results in a discontinuity in the image[7]. There are three main types of discontinuities in the images based on isolated points, lines and edges. Derivatives are mostly used to detect the discontinuity in an image. For isolated points detection Laplacian operator or thresholding can be used. Spatial masks like Sobel, prewitt, Roberts, etc. can be used to detect all the three types of discontinuities in an image. Most common operators used for edge detection are Roberts's edge detection, Kirsch edge detection, Robinson edge detection, Marr-Hildreth edge detection, LoG edge detection and Canny Edge Detection [8]. 


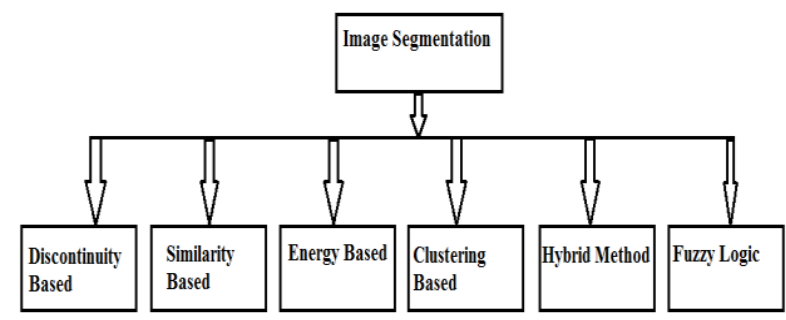

Fig 1:Image Segmentation Methods Classification

\subsection{Similarity based methods}

In similarity based approach region is an important concept in interpreting an image because regions may correspond to objects in the image. Partitioning into regions is done by using gray values of pixels. The pixels that correspond to an object are grouped and marked. The common procedure is to compare one pixel with its neighbors. If a similarity criterion is satisfied, the pixel can be set belong to the cluster as one or more of its neighbors. Seed based region growing [9] Region growing techniques work well in noisy images where edges are difficult to detect. This method group pixels or sub regions into larger regions based on certain criteria. In this method one seed point is selected and then 8 neighboring points of this seed point are selected and then compared on the basis of chosen criteria. Then pixel aggregation is done by using seed points in a way that corresponding regions grow by adding to each seed point those neighboring points that have some similar properties [10].Fast scanningis like a unseeded region growing algorithm the number of clusters is not decided before getting the image. It is very fast and simple method. The Image is scanned from upper left to lower right corner by this method and it checks whether the pixel can be merged into an existing cluster or not. A threshold value is fixed beforehand. The difference between the pixel value and average intensity value of neighboring cluster smaller than threshold (fixed before) then pixel is merged into the cluster. Although it does not provide a global view of the problem, also causes the gradient problem and very sensitive to noise.It takes lesser time than other segmentation algorithms and provides good shape matching with real world objects.Dynamically Growing Hierarchical self organizing map[11] is also giving better results in similarity based segmentation of images.

\subsection{Energy based methods}

The popular Snakes method was proposed by Kass et.al[12] in this method a curve evolves under some force till it stops at the boundary. The curve moves to minimize the energy. This method uses a framework in which local minima of energy functional is having the set of alternate solutions. By adding the suitable energy terms to the minimization it is possible for the user to push the model out of local minima towards the desired solution. The result is an active model that falls into desired solution when placed near it. The snake model proposed by Kass is active and is always minimizing its energy functional and therefore exhibits dynamic behavior. This method is popular under the class of parametric Active contour models. Active contour energy based method can be categorizedas parametric and geometric.The Parametric Active contour methoduses parameterized curves to represent the contours. The model requires a constant curve to detect the boundary of the image[13]. These contours are formulated by minimizing energy functional. This minimization function has both the internal and external forces also called internal and external energies. Internal energy defines geometric properties of a curve and external energy consists of all other forces that guide the curve to delineate to the desired contour. Many types of external and internal forces have been defined so far like few external forces are pressure force, Gaussian potential force.Geometric Active contours [15] are based on the theory of curve evolution and the level set method. In this framework curves evolves using geometric properties only and not use curve's parameters. This approach allows automatic changes in the topology of active contour such as in contours merging and splitting during evolution, when implemented using level set methods. In geometric active contour methods effect of normal force is taken as tangential forces effects parametric methods only. Geometric active contours can be categorized as edge based models and region based models[16]. Edge based methods use image gradient force to attract.Mumford Shah method: Mumforddescribed the segmentation process as dividing the image into homogeneous regions separated by smooth boundaries and finding the minimum of a function made from combining length of boundary, the gradient of a smoothed version of the image and difference of true and smoothed images. In this method Euler equations are used for boundaries along with hill climbing. In this method first time piecewise smooth formulation was proposed. These piecewise smooth models can handle the intensity inhomogeneity problems of segmentation. Various reformulations of region based methods are used for segmentation like Local region based active contour method reformulated the segmentation energy in a local way.

\subsection{Clustering based methods}

It is a kind of unsupervised learning job, no training stages are used in clustering instead they train themselves by means of existing information. It is used when classes are already identified. Clustering is a categorization of objects in groups according to certain belongings of these objects.Clustering methods, aims to find out vector from local image regions. There are different types of clustering like: $\mathrm{K}$ means clustering, C means Clustering, mountain clustering method and subtractive clustering method.K-means clustering is simple and computationally faster technique and also faster than hierarchical techniques[16]. The number of clusters and centroid are chosen at first very carefully as this method will give different results based on this selection. This method mainly works in two phases. In the first phase it calculates $\mathrm{k}$ the centroid and in a second phase it takes each point to cluster which has nearest centroid from the respective data point. To calculate the nearest centroid distance various methods have been used, e.g. Euclidean distance. Once the grouping of clusters is done the new centroid of each cluster is calculated and further Euclidean distance between each center and each data point. The points with minimum Euclidean distance are assigned in a cluster. This is an iterative method that minimizes the sum of distances from each object to its cluster centroid over all clusters.Although Fuzzy C-Means and $\mathrm{K}$ Means are efficient techniques but they are time consuming.Thedynamically Growing Hierarchical Self Organizing Map (DGHSOM) [11,17] overcomes the problem of specifying the number of clusters and total number of iteration using quality threshold clustering which divides data and having more computing power than $\mathrm{k}$-means.

\subsection{Hybrid methods}

These methods combine one or more existing segmentation methods. These methods rely on morphological operations performed on the images. For example: Local binary fitting(LBF) local intensity fitting(LIF)[18], Local Gaussian 
Intensity fitting(LGIF), Local region based active contour method(LRBAC)[19].Local Binary Fitting model [18] by embedding the local image information. It also works well on images with intensity inhomogeneity. The main idea in this method is to add a kernel function to define the energy functional.

$\mathrm{E}^{\mathrm{LBF}}(\mathrm{C}, \mathrm{f} 1, \mathrm{f} 2)=\lambda 1 \int_{\Omega} \int_{\text {inside }(\mathrm{C})} \mathrm{K}_{\sigma}(\mathrm{x}-\mathrm{y})|\mathrm{I}(\mathrm{y})-\mathrm{f} 1(\mathrm{x})|^{2} \mathrm{dx}$ $\mathrm{dy}+\lambda 2 \int_{\Omega} \int_{\text {outside(c) }} \mathrm{K}_{\sigma}(\mathrm{x}-\mathrm{y})|\mathrm{I}(\mathrm{y})-\mathrm{f} 2(\mathrm{x})|^{2} \mathrm{dy} \mathrm{dx}, \mathrm{x}, \mathrm{y} \epsilon \Omega$

Where $\lambda 1$ and $\lambda 2>0$ are fixed parameters, $K_{\sigma}$ is Gaussian kernel with standard deviation $\sigma$, f1 and $\mathrm{f} 2$ are two smooth functions that approximate the local image intensities inside and outside the contour.The standard deviation of kernel plays an important role, it can be observed as scale value that controls, region scalability from small neighborhood of the whole image domain. It is always carefully chosen as not too small and not too large to get better results.Here $\mathrm{f} 1(\mathrm{x})$ and $\mathrm{f} 2(\mathrm{x})$ are the averages of image intensity inside and outside the contour. This is the reason why this method can handle the problem of intensity inhomogeneity well.

Local Image fitting method is a modified form of the above method. This method also takes care of intensity inhomogeneity problem, but get stuck in local minima sometimes. LIF energy attracts the contour towards object boundaries and mainly dominant near object boundaries. This method can be viewed as constraint of difference between fitting and original image[20]. In this model a truncated Gaussian window is taken $\mathrm{K}_{\sigma}(\mathrm{x})$ with standard deviation $\sigma$ and size of $4 \mathrm{k}+1$ by $4 \mathrm{k}+1$ where $\mathrm{k}$ is greater integer smaller than $\sigma$. Although this method is good for intensity inhomogeneity problem, but re initialization was expensive and time consuming. Traditional level set formulation of this method used signed distance function to its interface and during evolution as re initialization is necessary to restore degraded level set function. To regularize the level set function Gaussian kernel is used in this method.This method can be implemented without re initialization that is by using the Euclidean length term that regularize the level set function. This will remove the initialization need, but it gets trapped in local minima. On the whole this method is more computationally efficient than LBF.

Local Gaussian intensity fitting: Li et al.[21] proposed a Local Gaussian distribution of energy with level set functions, local means and variances.These means and variances of local intensities are considered as spatially varying functions. These methods take care of images with intensity inhomogeneity problem. And moreover, it can be applied on 3D data as well. This method is a region based active contour method with variational level set formulation, in which a kernel function is used to define the energy to characterize the fitting of local Gaussian distribution to local image data around the neighborhood of a pixel. Then the double integral of this is calculated over the entire image to form internal energy. While calculating the resultant that minimizes the energy functional, the local intensity information is used to find variances and therefore guide them option of contour. And so it handles images with intensity inhomogeneity also.

Local region based Active contour method:Lankton et al. [22]proposed a framework for region based segmentation energy to be formulated in a local way. As most of the medical and natural images are heterogeneous in nature that is objected to be segmented cannot be distinguished on the terms of global intensities which can further lead to error prone segmentation. So to counter this problem Lankton etal. proposed a new class of active contours energies which considers local information plus the benefits of region based techniques. LRBAC provide objectives like it can be used to localize any region based energy, it provided a way to localized active contour to interact and it provides a way by which any region based energy can be localized in a local way.This method basically divides the whole image into local smaller regions and in turn creates local energies at each point along the curve. In this method three energies that come intothe picture are uniform modelling energy, means separation energy and histogram separation energy. These energies interact simultaneously to segment multiple objects. So this method allows localized active contours to compete in an image while segmenting different objects that may or may not share borders.

Implicit Active contour model with local and global intensity fitting energies method integrates the Local intensity fitting energy with an auxiliary global intensity fitting energy. The LIF energy attracts the contours near the edges of objects or boundaries. GIF incorporates global image information to improve the robustness to initialization of the contours. As compared to previous LIF, LRBAC [23] provides accurate segmentation results regardless of initialization. This method is capable of finding objects with interior holes or blurred edges also. It also handles intensity inhomogeneity efficiently while allowing more flexible initialization and maintains the sub pixel accuracy.Medical Image Segmentation Based on a Hybrid Region-Based Active Contour Modelis a new hybrid method based on Active contour model to segment the images with intensity inhomogeneity. The energy functional in this method comprises of three weighted terms Local, global and regularization term. The total energy is incorporated in a level set formulation with a level set regularization term from which a curve evolution equation is derived for energy minimization.This is based on the region information of the images and use both global and local information to attain the correct result quickly[24].The global information is provided by $\mathrm{CV}$ model. The local information is described by applying the framework of the energyin the localizing the energy. The global term in energy functional will take care of images with weak boundaries, Local term will take care ofimages with intensity inhomogeneity and to stop the curve from weaving around areas of noise regularization term is added. The energy function for this model is non-convex and therefore has local minima which makes it sensitive to initialization of contour.

\subsection{Fuzzy logic based methods}

Fuzzy image processing approach process the images, their segments and features as fuzzy sets. Each fuzzy set has one membership function defined on all image pixels. Each image pixels can be part of various membership functions. These methods use fuzzification function to remove noise from the image and create a fuzzy image. Fuzzy means unclear or undefined. After some membership modification using fuzzy logics image is defuzzified to get the results. There are various methods based on fuzzy logic that are used for segmentation like Fuzzy C means Clustering. It is an unsupervised clustering method that enables objective comparison of various methods without human intervention [25]. Clustering can be of different types like hard clustering and soft clustering. In many real time images some pixels can belong to more than one cluster or there can be some membership degree of pixels to some clusters. So to it leads to fuzzy belongingness and Fuzzy $\mathrm{C}$ Means clustering method come into light. In an image neighboring pixels are highly correlated and possess same intensity values so spatial 
relationship is important while clustering. Spatial information is used to remove the noise.

FCM is an iterative algorithm. It generates a partition matrix and objective function. Cluster center and objective function values remain changing after every iteration. The traditional FCM is sensitive to noise and Shamsi et al. [26] proposed a modified FCM that incorporates spatial information along with traditional FCM and modifies membership weighting of each cluster, every point of data set has a weight in relation to every cluster.This weight permits to have a better classification in case of noise.Ji. $\mathrm{Z}$ et al [27] proposed another modified possiblistic $\mathrm{C}$ means of images that have been corrupted by intensity inhomogeneity and noise. They introduced a method to compute the weight of local spatial in the objective function and their algorithm is capable of utilizing local contextual information to impose spatial continuity which in turn helps in noise removal. To deal with intensity inhomogeneity global intensity is introduced. Compared to other methods it gives better performance, It is robust to initialization and thus used for fully automatic applications.

\section{TESTS AND OUTCOME ANALYSIS}

Microarray Image segmentation using Improved Globally optimal geodesic active contour[28] has proved that this gives better results than the actibve contour method and other geodesic method.In this part, we compare the energy based methods the first one was active contours without edges by $\mathrm{T}$. Chan and L. Vese [29] regarding image segmentation, the second one ended up being Active contours according to the local Gaussian distribution presented by Wang [30], the third one ended up being Active contours driven by local image fitting energy introduced by Huihui Song [31], plus the last one was A Level Set Method in the Presence of Intensity Inhomogeneities presented by Chunming $\mathrm{Li}$ [32].There are so many filters proposed for removal of noises in the medical image in this category LHM Filter for Removal Salt and Pepper with Random Noise[33] is giving better results for noisy medical images. Fig. 2 shows the results for medical image in which initial contours are plotted, from then on curve progression steps are presented soon after sufficient progressed, we got a region of interest and the approach can be revealed from the Fig. 2
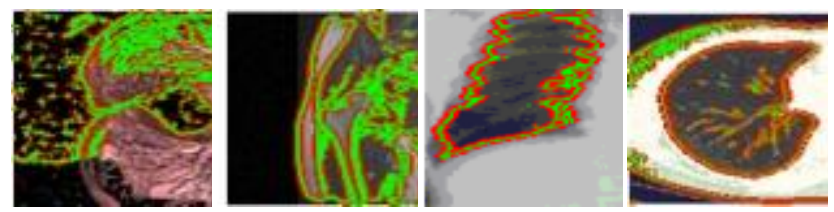

(a)
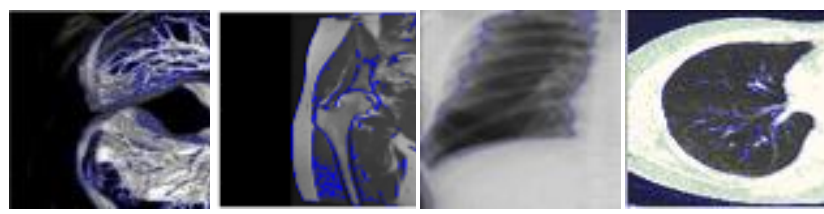

(b)
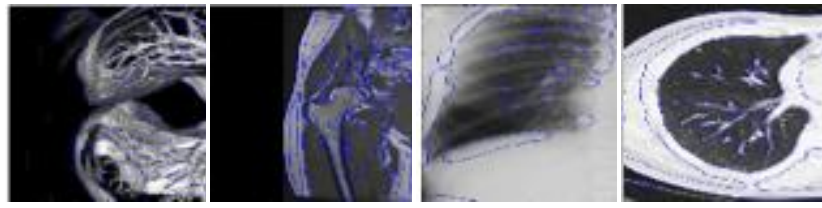

(c)
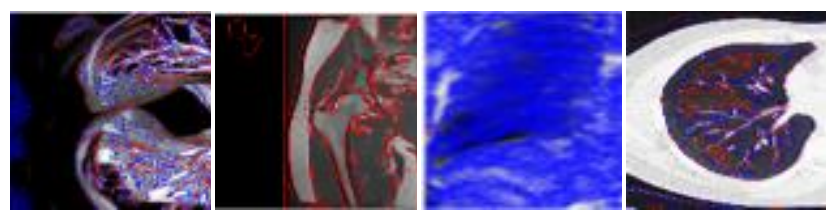

(d)
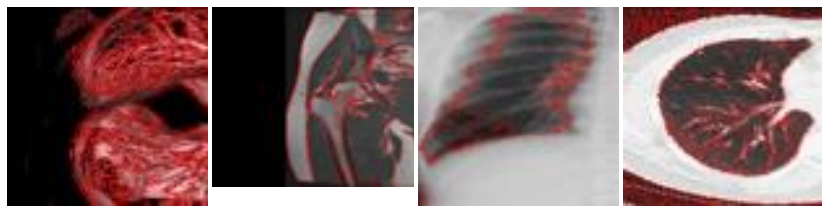

(e)

Fig 2: Shows the segmentation result of medical MRI and

CT images of the human body. Figures are showing segmentation results Fig 2(a) using the active contour method without edges, Fig. 2(b) using the improved Chan Vese method, Fig. 2(c) using the Active contour method by local image fitting,Fig. 2(d) shows the segmentation result using the A Level Set Method for Image Segmentation in the Presence of Intensity Inhomogeneities with

Application to MRI,Fig. 2s(e) shows the energy based method.

A review of energy based methods like active contour, level set, local binary fitting, local intensity fitting etc. of the prior literature relevant to the research conducted is mentioned in this paper. The following research areas have been addressed: intensity inhomogeneity, incorrect segmentation and slow processing in the medical image segmentation. In particular, intensity inhomogeneity often seen in medical images, such as microscopy, computer tomography (CT), ultrasound, and magnetic resonance imaging (MRI). For example, the intensity inhomogeneity in MRI often appears as an intensity variation across the image, due to radio frequency (RF) coils or acquisition sequences. In addition, images are often corrupted by various noises that challenge the segmentation. We also present the review of incorrect and slow processing of the energy based models and challenges of the methods. High noise level set methods are one of the methods which are pretty good and can deal with the noise problem. Experimental results demonstrated that energy based model possesses better performance than the $\mathrm{CV}$ model and LIF model and also confirmed its effectiveness in various synthetic images and actual images. Experimental results display its advantages regarding accuracy, proficiency, and robustness. The local binary fitting (LBF) and Improved energy based are more accurate and efficient segmentation methods. 
Table 1.Comparison ofcomputation time of all the energy based methods

\begin{tabular}{|c|r|r|r|c|}
\hline \multirow{2}{*}{ Methods } & \multicolumn{4}{|c|}{ CPU Time(s) } \\
\cline { 2 - 5 } & $\mathbf{1}$ & $\mathbf{2}$ & $\mathbf{3}$ & $\mathbf{4}$ \\
\hline Energy Based & 6.073 & 5.220 & 5.668 & 11.747 \\
\hline Chan Vese & 9.179 & 15.453 & 18.038 & 16.496 \\
\hline $\begin{array}{c}\text { Improved Chan } \\
\text { Vese }\end{array}$ & 8.270 & 4.525 & 14.556 & 12.723 \\
\hline $\begin{array}{c}\text { Local image } \\
\text { fitting }\end{array}$ & 9.851 & 17.364 & 36.987 & 32.966 \\
\hline Level Set Method & 10.531 & 10.796 & 49.453 & 49.750 \\
\hline
\end{tabular}

As evident from the results that traditional level set method takes too much time for computation. Local image fitting method is faster than Level set methods but takes more time than Chan Vese method. Improved version of Chan Vese is better than the traditional Chan Vese method as former is taking lesser time. Also in certain cases the segmentation time depends on the type of image that is features of image like patterns, edges etc. But out of all the methods Energy based methods are giving much better results than other methods irrespective of features of images.

\section{CONCLUSION}

Theenergy based method gives better performance than CV method, Improved CV, An Active contour method by local image fitting energy, Level Set Method with bias correction. It takes less time than other methods it can be seen how the energy based approach gives better result compared to ChanVese, Enhanced Chan-Vese, Local image fitting energy method and Level Set Method Intensity Inhomogeneities whatever the shape and the position of the initial curve. The consequent changes with the entire initial contour and you will need to run the method so you can choose the best result; hence, this method cannot be used, intended for accurate and proper segmentation, CV model uses location information and considered as one of the most used models intended for segmentation. One of many prominent aspects of the $\mathrm{CV}$ model is which it performs well for the images having fuzzy without ends. However, being a limitation, your CV model always supposes image having intensity homogeneity, the local image fitting process gives appropriate result.

\section{REFERENCES}

[1] Kryjak, T. (2014). Foreground Object Segmentation in Dynamic Background Scenarios.Image Processing \& Communications, 19(2-3). http://dx.doi.org/10.1515/ipc2015-0007

[2] Miao, Y. \& Shi, W. (2012). Level Set Segmentation Method in Medical Image Segmentation Research and Application. AMM, 157-158, 1012-1015. http://dx.doi.org/10.4028/www.scientific.net/amm.157158.1012

[3] Lew, M., Sebe, N., Djeraba, C., \& Jain, R. (2006). Content-based multimedia information retrieval. ACM Trans. Multimedia Comput. Commun. Appl., 2(1), 1-19. http://dx.doi.org/10.1145/1126004.1126005

[4] Pham, D., Xu, C., \& Prince, J. (2000). Current Methods in Medical Image Segmentation1.Annual Review Of
Biomedical Engineering, 2(1), 315-337. http://dx.doi.org/10.1146/annurev.bioeng.2.1.315

[5] Krstinić, D., Skelin, A., \& Slapničar, I. (2011). Fast twostep histogram-based image segmentation. IET Image Process., 5(1), 63. http://dx.doi.org/10.1049/ietipr.2009.0107

[6] Kezia, S., Prabha, I., \& Kumar, V. (2013). A ColorTexture Based Segmentation Method To Extract Object From Background. International Journal Of Image, Graphics And Signal Processing, 5(3), 19-25. http://dx.doi.org/10.5815/ijigsp.2013.03.03

[7] Salman, N., Ghafour, B., \& Hadi, G. (2015). Medical Image Segmentation Based on Edge Detection Techniques. Advances In Image And Video Processing, 3(2). http://dx.doi.org/10.14738/aivp.32.1006

[8] sha, N., Mehra, R., \& Sharma, L. (2015). Comparative Analysis of Canny and Prewitt Edge Detection Techniques used in Image Processing. IJETT, 28(1), 4853. http://dx.doi.org/10.14445/22315381/ijett-v28p210

[9] Alidoost, F., Sharifi, M., \& Stein, A. (2015). Region- and pixel-based image fusion for disaggregation of actual evapotranspiration. International Journal Of Image And Data Fusion, 6(3), 216-231. http://dx.doi.org/10.1080/19479832.2015.1055834

[10] Rajkumar, K. \& Raju, G. (2015). Automated Mammogram Segmentation Using Seed Point Identification and Modified Region Growing Algorithm. British Journal Of Applied Science \& Technology, 6(4), 378-385. http://dx.doi.org/10.9734/bjast/2015/14383

[11] N. Waoo, R. Kashyap and A. Jaiswal, 2010,DNA Nano array analysis using hierarchical quality threshold clustering, The 2nd IEEE International Conference onInformation Management and Engineering (ICIME), Chengdu, 2010, pp. 81-85.doi: 10.1109/ICIME.2010.5477579

[12] Kass, M., Witkin, A., \& Terzopoulos, D. (1988). Snakes: Active contour models. Int J Comput Vision, 1(4), 321331. http://dx.doi.org/10.1007/bf00133570

[13] Moallem, P., Tahvilian, H., \& Monadjemi, S. (2015). Parametric active contour model using Gabor balloon energy for texture segmentation. Signal, Image And Video Processing, 10(2), 351-358. http://dx.doi.org/10.1007/s11760-015-0748-6

[14] Koibuchi, H. \& Shobukhov, A. (2016). Internal phase transition induced by external forces in Finsler geometric model for membranes. Int. J. Mod. Phys. C, 27(04), 1650042. http://dx.doi.org/10.1142/s012918311650042x

[15] CAO, J., ZENG, Q., \& WANG, J. (2011). Particle filter tracking algorithm based on geometric active contours. Journal Of Computer Applications, 31(5), 1205-1208. http://dx.doi.org/10.3724/sp.j.1087.2011.01205

[16] Zhou, S., Xu, Z., \& Tang, X. (2010). Method for determining optimal number of clusters in Kmeans clustering algorithm. Journal Of Computer Applications, 30(8), 1995-1998. http://dx.doi.org/10.3724/sp.j.1087.20

[17] D.Patil, D. \& Gupta, P. (2015). Growing Hierarchical Self-Organizing Map (GHSOM) for Mining Gene Expression Data. International Journal Of Computer 
Applications, $\quad 109(2), \quad 16-17$. http://dx.doi.org/10.5120/19160-0603

[18] Zhao, S., Zhou, M., \& Wang, K. (2013). A Statistical Local Binary Fitting Model for Blood Vessel Segmentation. AMR, 756-759, 3430-3434. http://dx.doi.org/10.4028/www.scientific.net/amr.756759.3430

[19] Wang, L., He, L., Mishra, A., \& Li, C. (2009). Active contours driven by local Gaussian distribution fitting energy. Signal Processing, 89(12), 2435-2447. http://dx.doi.org/10.1016/j.sigpro.2009.03.014

[20] Peng, Y., Liu, F., \& Liu, S. (2013). Active contours driven by normalized local image fitting energy. Concurrency And Computation: Practice And Experience, 26(5), 1200-1214. http://dx.doi.org/10.1002/cpe.3053

[21] Wang, L., He, L., Mishra, A., \& Li, C. (2009). Active contours driven by local Gaussian distribution fitting energy. Signal Processing, 89(12), 2435-2447. http://dx.doi.org/10.1016/j.sigpro.2009.03.014

[22] Lankton, S. \& Tannenbaum, A. (2008). Localizing Region-Based Active Contours. IEEE Transactions On Image Processing, 17(11), 2029-2039. http://dx.doi.org/10.1109/tip.2008.2004611

[23] Xu, X. \& He, C. (2013). Implicit Active Contour Model with Local and Global Intensity Fitting Energies. Mathematical Problems In Engineering, 2013, 1-13. http://dx.doi.org/10.1155/2013/367086

[24] Liu, T., Xu, H., Jin, W., Liu, Z., Zhao, Y., \& Tian, W. (2014). Medical Image Segmentation Based on a Hybrid Region-Based Active Contour Model. Computational And Mathematical Methods In Medicine, 2014, 1-10. http://dx.doi.org/10.1155/2014/890725

[25] Zaixin, Z., Lizhi, C, \& Guangquan, C. (2014). Neighbourhood weighted fuzzy c-means clustering algorithm for image segmentation. IET Image
Processing, 8(3), 150-161. http://dx.doi.org/10.1049/ietipr.2011.0128

[26] Shamsi, H. \& Seyedarabi, H. (2012). A Modified Fuzzy C-Means Clustering with Spatial Information for Image Segmentation. IJCTE, 762-766. http://dx.doi.org/10.7763/ijcte.2012.v4.573

[27] Ji, Z., Sun, Q., \& Xia, D. (2011). A modified possibilistic fuzzy c-means clustering algorithm for bias field estimation and segmentation of brain MR image. Computerized Medical Imaging And Graphics,35(5), 383-397.

http://dx.doi.org/10.1016/j.compmedimag.2010.12.001

[28] Kashyap, R. \& Gautam, P. (2013). Microarray Image Segmentation Using Improved GOGAC Method. International Journal of Computer Science And Engineering, 2(4), 67-74.

[29] Chan, T. \& Vese, L. (2001). Active contours without edges. IEEE Transactions On Image Processing, 10(2), 266-277. http://dx.doi.org/10.1109/83.902291

[30] Wang, L., He, L., Mishra, A., \& Li, C. (2009). Active contours driven by local Gaussian distribution fitting energy. Signal Processing, 89(12), 2435-2447. http://dx.doi.org/10.1016/j.sigpro.2009.03.014

[31] Zhang, K., Song, H., \& Zhang, L. (2010). Active contours driven by local image fitting energy. Pattern Recognition, 43(4), 1199-1206. http://dx.doi.org/10.1016/j.patcog.2009.10.010

[32] Chunming Li, Rui Huang, Zhaohua Ding, Gatenby, J., Metaxas, D., \& Gore, J. (2011). A Level Set Method for Image Segmentation in the Presence of Intensity Inhomogeneities With Application to MRI.IEEE Transactions On Image Processing, 20(7), 2007-2016. http://dx.doi.org/10.1109/tip.2011.2146190

[33] Prasad, V. \& Gopal, R. (2016). LHM Filter for Removal Salt and Pepper with Random Noise in Images. International Journal Of Computer Applications, 139(1), 9-15. http://dx.doi.org/10.5120/ijca2016908962 